\title{
Invited reply
}

\section{The uncertainties of the largest fossil rodent}

Millien (2008) gave a technical contribution to the estimation of the largest fossil rodent's body mass. A very relevant conclusion is that fosephoartigasia monesi does not fit any extant rodent model. Interestingly, Millien (2008) established that $\mathcal{F}$. monesi had a very elongated skull or a very short tooth row. In a first approach to the problem, we assumed that the tooth row was very short and left aside this measurement for the body mass estimation (Rinderknecht \& Blanco 2008). At present, I agree with Millien's (2008) general comment on this point: it is not possible (without independent measures) to leave aside tooth row (or skull length). Nevertheless, in this case we made our choice based on the study of the closest living relative. Prior to the body mass estimation of F. monesi, we performed a test of the allometric equations method. We estimated the body mass of the only living species of the Dinomyidae family: Dinomys branickii from many cranial measurements. The results were very close to the measured body mass for almost all the cranial measurements considered the first time. However, two measurements give a serious underestimation of the body mass: tooth row length and grinding tooth total surface. The results of Millien (2008) are consistent with our observations: the lowest value of body mass was from tooth row length. A direct observation of the skull of D. branickii is enough to suggest that its tooth row is very small. But the case for $\mathcal{F}$. monesi is unknown as Millien (2008) stated. There are allometric effects of rostral length in rodents (Lessa \& Patton (1989) and references therein) and f. monesi goes even beyond the predictions of these models as Millien (2008) shows in fig. 1.

Millien (2008) also emphasized that body mass estimations should be considered with caution. For that reason, we made our first estimation following Reynolds (2002), a very relevant paper on the topic of methodological considerations on rodent's body mass estimation. The estimation, following Reynolds (2002) gives us extreme values of 716 and $2250 \mathrm{~kg}$ (Rinderknecht \& Blanco 2008). Millien (2008) did not specifically raise any criticism on this method as applied in Rinderknecht \& Blanco (2008). We also added to our manuscript a first approach to body mass estimation by using more closely related living rodents. Our sample was small because we were only attempting a first approach to the problem and the available data at the Museo Nacional de Historia Natural y Antropología did not have body mass records for all the specimens. The fact that Millien (2008) provided a much better sample of rodents is useful, but unfortunately Millien recalculated the allometric model based only on data in Rinderknecht \& Blanco (2008). The best sample was used only for upper tooth measurements. Our technical conclusions (leaving aside all the media

The accompanying comment can be viewed on page 1953 or at http:// dx.doi.org/10.1098/rspb.2008.0087. simplifications and speculations about body length) is that 7. monesi's body mass was probably between 468 and $2586 \mathrm{~kg}$, a very broad range of error. However, Millien's (2008) first paragraph highlighted only our largest estimations, ' $\mathcal{~}$. monesi was estimated to have weighed $1211 \mathrm{~kg}$ on average, and perhaps as much as $2584 \mathrm{~kg}$ '. The mean value obtained by Rinderknecht \& Blanco (2008) was $1211 \mathrm{~kg}$, and I recognize that this value does not mean much. Only a rough estimation is possible. Only some results obtained by Millien (2008) are smaller than those of Rinderknecht \& Blanco (2008), for many anatomical measurements Millien's results are larger than those of Rinderknecht \& Blanco's. The mean value of Millien (2008) is approximately $900 \mathrm{~kg}$; it is a general picture very close to the results of Rinderknecht \& Blanco (2008). In a problem with such large uncertainties, a $25 \%$ of difference in values is not a big one. I think that her methods probably are finer but our conclusions are very similar. Unfortunately, the final claim in Millien (2008) highlights only one aspect, 'body mass may have been as low as $350 \mathrm{~kg}$. The statement is right, but from her results it can be said that body mass may have been also as large as $1534 \mathrm{~kg}$. I think the best way to tackle error sources in science is to be as objective as possible about results in technical communications. I think that being cautious with sources of errors means (among many technical features) not going beyond results and methods when we make final conclusions. We took into account the difficulties by using two different methods and providing the maximum range of variation (from 468 to $2686 \mathrm{~kg}$ ).

A minor criticism to Millien (2008) is that measurements must be taken from the original material and not from the published pictures if possible. The measurements themselves can be a source of errors. In a problem with so many uncertainties, this source of error could perhaps be neglected. In any case, for further consideration of methods, the way that the measurements were taken must be addressed. Many cranial measurements of F. monesi appeared in Rinderknecht \& Blanco (2008), but Millien (2008) added three new measurements probably from the pictures. The method used to obtain all the measurements and values must be included in these kinds of works or at least in supplementary data.

Finally, there are two issues in Millien (2008), which place doubt on her results. The estimation of $272 \mathrm{~kg}$ from incisors width obtained by Millien (2008) is extremely different from our estimation. Such an extremely low value obtained by Millien (2008) is counterintuitive because the incisors of f. monesi are not extraordinarily reduced. This is even stranger because a closely related estimation based on upper incisors transverse diameters presented only in Millien (2008) gives a body mass of $650 \mathrm{~kg}$. Even the small tooth row length gives in Millien (2008) a larger body mass estimation of $356 \mathrm{~kg}$. Two colleagues 
recalculated independently this and other values of Rinderknecht \& Blanco (2008). One of them (Washington W. Jones 2008, personal communication) made a deep study of the case exploring variations in the allometric exponent also by using reduced major axis methods (not only least squares as we did) or by using rounded values of log-transformed data. He used different statistical software, but never obtained a value close to 2.52 as in Millien (2008). A possible explanation would be that Millien (2008) used only interspecific data, and this explains why her results are very different. However, we recalculated from the interspecific supplementary data of Millien (2008) and obtained allometric exponents of approximately 3.49 (smaller than our 3.9 value) but not a value close to 2.52 . Some recalculations from other authors are needed to clarify the source of the error.

The other issue is about our regression method. Millien (2008) stated that Rinderknecht \& Blanco (2008) did not log transform all data before regression. This was not the case and there are no words in Rinderknecht \& Blanco (2008) consistent with Millien's (2008) claim.

Millien (2008) improved some points of our first approach. But Rinderknecht \& Blanco (2008) and Millien's (2008) results are very close. It is not possible to exclude body masses of approximately $1000 \mathrm{~kg}$ (suggested by Rinderknecht \& Blanco 2008) based only on the results obtained by Millien (2008). New methods or fossil findings would improve our present status of very large uncertainties on this problem. In any case, we must remember that body mass estimation from only a skull of an extinct species with such a large difference in size to the largest living relatives is a very difficult problem. Large uncertainties are always present and more precise measurement methods are not necessarily more accurate but using better methods is always encouraged.

I thank María José Salerno for improving the English language of the manuscript, Washington W. Jones for his independent recalculation of the results of Rinderknecht \& Blanco (2008) and Millien (2008) and Andrés Rinderknecht for his useful comments.

$$
\begin{array}{r}
\text { R. Ernesto Blanco* } \\
\text { Facultad de Ingeniería, Instituto de Física, fulio Herrera y } \\
\text { Reissig 565, Montevideo 11300, Uruguay } \\
\text { *blanco@fing.edu.uy }
\end{array}
$$

\section{REFERENCES}

Lessa, E. P. \& Patton, J. L. 1989 Structural constraints, recurrent shapes, and allometry in pocket gophers (genus Thomomys). Biol. F. Linn. Soc. 36, 349-363. (doi:10.1111/ j.1095-8312.1989.tb00500.x)

Millien, V. 2008 The largest among the smallest: the body mass of the giant rodent fosephoartigasia monesi. Proc. $R$. Soc B 275, 1953-1955. (doi:10.1098/rspb.2008.0087)

Reynolds, P. S. 2002 How big is a giant? The importance of method in estimating body size of extinct mammals. f. Mammal. 83, 321-332. (doi:10.1644/1545-1542(2002) $083<0321$ :HBIAGT $>2.0$. CO;2)

Rinderknecht, A. \& Blanco, R. E. 2008 The largest fossil rodent. Proc. R. Soc. B 275, 923-928. (doi:10.1098/rspb. 2007.1645) 